Musées, Patrimoine et Culture scientifiques et techniques

\title{
Un centre d'interprétation de l'Architecture et du Patrimoine pour le Pays de Montbéliard
}

\section{Gaëlle Cavalli}

\section{OpenEdition \\ Journals}

Édition électronique

URL : http://journals.openedition.org/ocim/359

DOI : 10.4000/ocim.359

ISSN : 2108-646X

Éditeur

OCIM

Édition imprimée

Date de publication : 1 septembre 2008

Pagination : $42-47$

ISSN : 0994-1908

Référence électronique

Gaëlle Cavalli, « Un centre d'interprétation de l'Architecture et du Patrimoine pour le Pays de

Montbéliard », La Lettre de l'OCIM [En ligne], 119 | 2008, mis en ligne le 21 janvier 2011, consulté le 30 avril 2019. URL : http://journals.openedition.org/ocim/359 ; DOI : 10.4000/ocim.359 


\section{Un centre d'interprétation de l'Architecture et du Patrimoine pour le Pays de Montbéliard}

\section{Gaëlle Cavalli *}

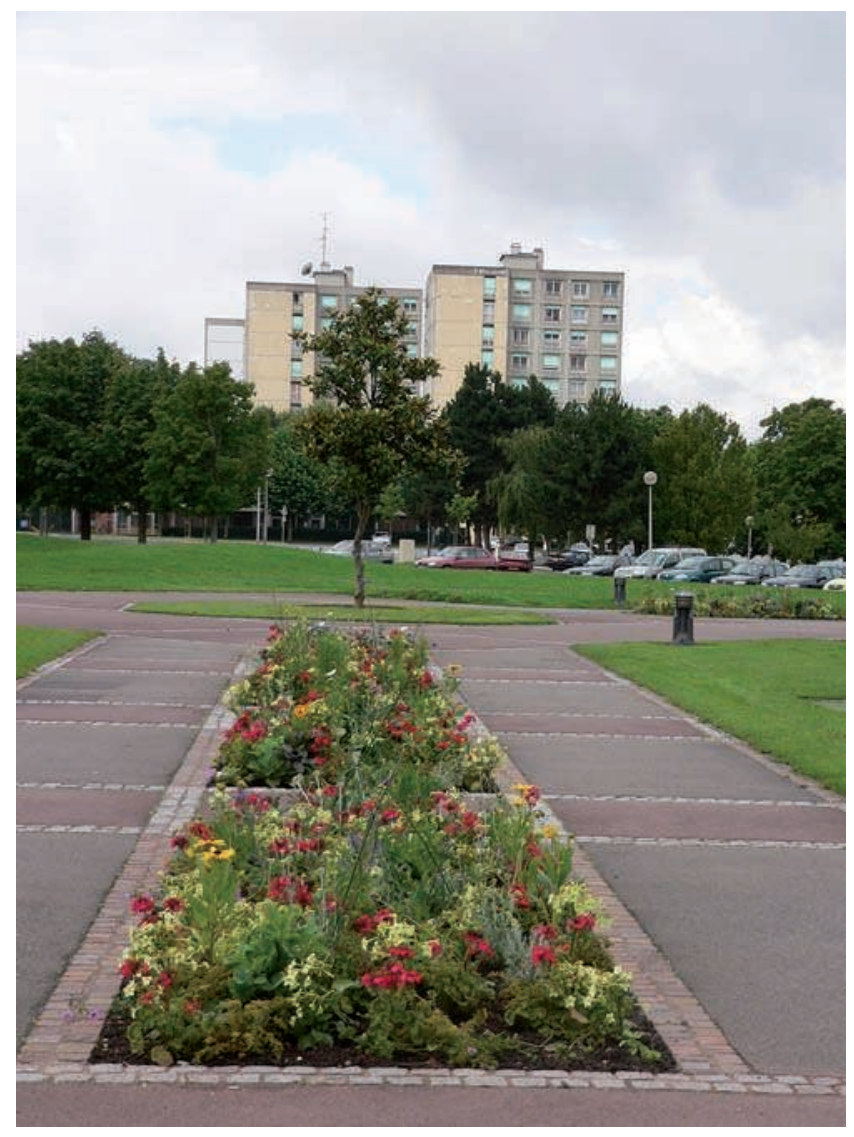

Le quartier de la Petite Hollande à Montbéliard, qui fait actuellement l'objet d'un programme de rénovation urbaine, pourrait être au cœur de la problématique développée par le centre d'interprétation de l'Architecture et du Patrimoine de Montbéliard.

() Évelyne Boilaux

* Gaëlle Cavalli est animatrice de l'Architecture et du Patrimoine, service Animation du Patrimoine, à la communauté d'agglomération du Pays de Montbéliard gaelle.cavalli@agglo-montbeliard.fr
Sur un territoire contrasté et multipolaire mais doté d'une forte identité historique, le projet du centre d'interprétation de l'Architecture et du Patrimoine du Pays de Montbéliard s'inscrit dans une démarche de consultation des habitants, du monde associatif et des élus qui devrait permettre de faire de ce lieu un lieu d'échange sur le patrimoine et un lieu de vie pour la population.

Le territoire de la communauté d'agglomération du Pays de Montbéliard est labellisé « pays d'Art et d'Histoire » par le ministère de la Culture et de la Communication depuis 1992. Un service baptisé «Animation du Patrimoine », créé en septembre 2006, en assure actuellement la gestion. Conformément à la convention qui lie la communauté d'agglomération au ministère de la Culture, une réflexion sur la création d'un centre d'interprétation d'Architecture et du Patrimoine répondant aux objectifs définis dans le document édité par le ministère est engagée (1).

Ce centre doit être un lieu d'information et de présentation des enjeux de l'évolution architecturale, urbaine et paysagère du pays :

- pour les habitants, un lieu de rencontre et d'information sur les activités de valorisation du patrimoine et les projets urbains et paysagers,

- pour les touristes, un espace d'information donnant les clés de lecture du pays,

- pour les jeunes, un support pédagogique dans le cadre des ateliers d'architecture et du patrimoine. Il comporte des espaces d'animation, un centre de documentation, des espaces d'exposition permanente et temporaire. 
Cette définition de ce que doit être un CIAP pour les villes et pays du réseau a en effet profondément évolué, notamment depuis les années 2000. Les premières « salles du patrimoine ", souvent considérées par les élus comme une obligation ou un simple plus dans des villes où l'offre patrimoniale est déjà abondante, étaient rarement au cœur d'une politique de développement touristique ou culturelle. On assiste aujourd'hui à une évolution de cette vision, où le CIAP peut être couplé à un musée par exemple.

\section{Ville et pays, deux histoires de territoire}

Parallèlement, une réflexion s'engage sur la traduction de ce type d'équipement, pensé au départ pour les villes d'Art et d'Histoire, pour les pays.

Deux cas de figure se rencontrent : le cas des labels « ville d'Art et d'Histoire » qui sont transformés en label pays (2) à la suite du transfert de la compétence à la communauté de commune ou d'agglomération, et d'autre part le cas de territoires ruraux, souvent extrêmement vastes (plus de 200 communes pour le pays d'Auxois par exemple).

Pour les premiers, la gageure est d'intégrer la spécificité des communes limitrophes de la grande ville dans le discours axé jusqu'alors sur la ville centre. La localisation du CIAP dans la ville centre et son unicité ne sont en général pas objet de débat.

En revanche, pour les pays correspondant à de vastes territoires, la mise en place du CIAP pose de nombreuses questions. Comment un seul lieu pourrait-il avoir un rôle dans l'aménagement du territoire, dans le sentiment d'appartenance des habitants à un pays, et sur quels critères choisir sa localisation ? Les réflexions s'orientent alors naturellement sur un CIAP décliné en plusieurs antennes. Mais comment justifier les coûts supplémentaires liés à la création de plusieurs équipements ? Et quel intérêt de créer de nouveaux équipements, alors que bien souvent des centres d'interprétation thématiques sont déjà en place sur le territoire?

Plusieurs pistes se dégagent : créer des pôles culturels permettant une mutualisation des moyens, où les antennes CIAP, thématiques, sont adossées à des bibliothèques par exemple (réflexion en cours dans le Pays de Riom). D’autre imaginent une dématérialisation du CIAP : la cohérence des divers équipements déjà en place serait assurée par la diffusion sur chacun d'eux d'un film ou support numérique donnant les clefs de compréhension globale du pays.

\section{L'exemple du Pays de Montbéliard : un centre pour des patrimoine variés}

Une autre piste est explorée pour le Pays de Montbéliard. Le cas de ce pays est en effet relativement original vis-à-vis des deux types décrits ci-dessus. Composé de 29 communes pour environ 122000 habitants, il ne s'agit pas d'un territoire rural, mais au contraire d'un territoire très urbanisé, même si cette urbanisation est émaillée de nombreux espaces naturels ou agricoles. Il ne dispose pas par ailleurs d'une ville centre à la taille disproportionnée comparée à celle des communes limitrophes. En effet, la ville de Montbéliard compte 28000 habitants, quand Audincourt et Valentigney en comptent respectivement 15600 et 12500 .

Ce territoire est donc contrasté et multipolaire. Il est également cohérent, car la communauté d'agglomération actuelle (faisant suite à l'un des plus anciens districts urbains de France, créé en 1959) correspond à un territoire assez proche de celui du Pays de Montbéliard historique marqué par la domination des Wurtemberg. Ce passé germanique et protestant - le Pays de Montbéliard est la seule région francophone d'obédience luthérienne -, lui a donné une identité forte, encore présente aujourd'hui, qui explique en grande partie le développement industriel qui a été le sien. Le Pays de Montbéliard est en effet le berceau de dynasties industrielles extrêmement importantes, comme les Japy et bien sûr les Peugeot.

D'autre part, la communauté d'agglomération dispose de sites patrimoniaux prestigieux, à ce jour peu mis en valeur, à l'exception du cœur historique de

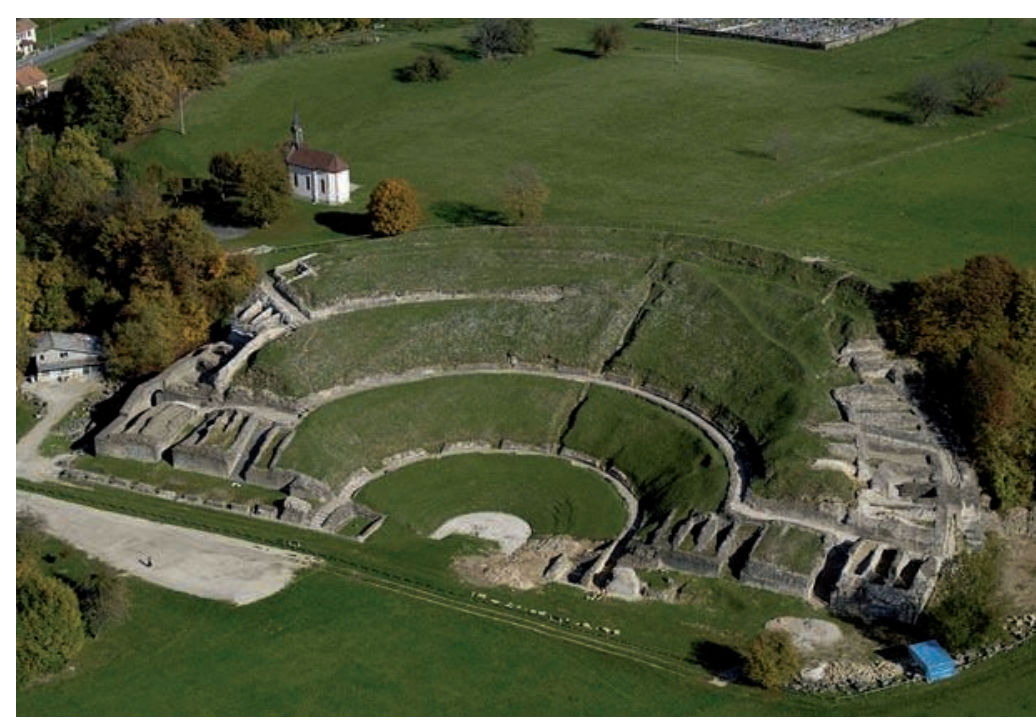

Le théâtre Gallo-romain de Mandeure (c) Claude Nardin 
Montbéliard et de son château, valorisés dans le cadre de la politique municipale. Ces sites font actuellement l'objet de projets de valorisation par la communauté d'agglomération.

Compte tenu de ces éléments, la réflexion s'est orientée vers la création d'un CIAP multipolaire, constitué d'une implantation principale qui serait située dans la ville centre, mais également d'antennes permettant de valoriser les principaux sites patrimoniaux tout en les replaçant dans l'histoire globale du pays. Le fil directeur proposé serait donc chronologique, mais également thématique.

À Mandeure, première antenne du CIAP, serait développé le patrimoine archéologique jusqu'à la fin de l'époque gallo-romaine. En effet, si le pays de Montbéliard dispose de quelques sites plus anciens, il prend son véritable essor aux époques gauloises et gallo-romaines. Grâce aux recherches récentes menées sur le site par des équipes internationales, l'importance du site de Mathay-Mandeure, dès l'époque gauloise, est aujourd'hui bien attestée. Les offrandes trouvées dans le sanctuaire gaulois constituent ainsi la plus importante collection conservée pour le monde celtique. Après la conquête romaine, Mandeure devient lieu du culte impérial. Ceci explique le considérable développement de la ville, ainsi que la qualité de l'architecture et des décors de ses monuments, notamment du théâtre, classé monument historique, le deuxième de Gaule par sa taille après celui d'Autun. La présence du théâtre, encore visible, et de l'équipe de recherche qui fait de cette agglomération antique très bien conservée une des mieux connues à ce jour en France expliquent l'intérêt de valoriser ce site d'importance nationale. Le projet de création d'un

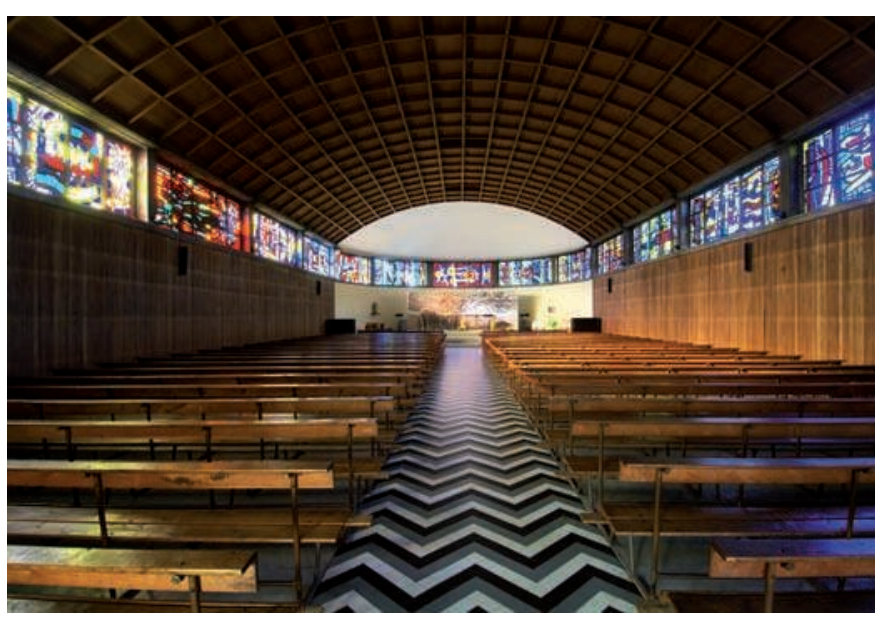

L'église du Sacré-Cœur d'Audincourt et ses vitraux de Fernand Léger (c) Claude Nardin pavillon est en cours d'étude. Il serait dédié à l'accueil des chercheurs et du public, au moyen d'espaces d'exposition temporaire, de conférences et d'animations, d'une galerie lapidaire présentant des blocs sculptés provenant du théâtre, d'un jardin romain et d'un espace d'initiation à la fouille. Un partenariat étroit avec le musée de Montbéliard, où sont conservées les collections issues du site, est prévu.

À Montbéliard, implantation principale du CIAP, seraient présentés le patrimoine alémanique et d'autre part le patrimoine des $\mathrm{XX}^{\mathrm{e}}-\mathrm{XXI}^{\mathrm{e}}$ siècles. Les périodes médiévales et modernes correspondent à un « âge d'or » du Pays de Montbéliard, et notamment de la ville, sous la domination des Wurtemberg. La communauté d'agglomération n'ayant pas vocation à se substituer à la ville de Montbéliard pour valoriser son patrimoine, l'action menée viendrait en complément des actions menées par la ville pour la mise en valeur de son château et de l'œuvre de l'architecte Heinrich Schickhardt (musées et sentier en centre ville par exemple). L'orientation donnée à l'exposition permanente serait donc plutôt l'évocation de l'influence alémanique sur le développement de la ville et du pays (évolution urbaine, architecture, protestantisme, mise en place d'un état moderne...). La présentation du patrimoine des $\mathrm{XX}^{\mathrm{e}}-\mathrm{XXI}^{\mathrm{e}}$ siècles serait également faite dans le CIAP de Montbéliard, et permettrait de présenter les projets d'urbanisme actuels, ce qui est une des vocations d'un CIAP. Les thèmes suivants pourraient être abordés : les grands ensembles des années 1950-1960, le dynamisme économique, les transformations urbaines actuelles (PLH, programmes ANRU...), les édifices religieux du XXe siècle, l'architecture civile contemporaine. La localisation précise de ce CIAP principal reste à déterminer.

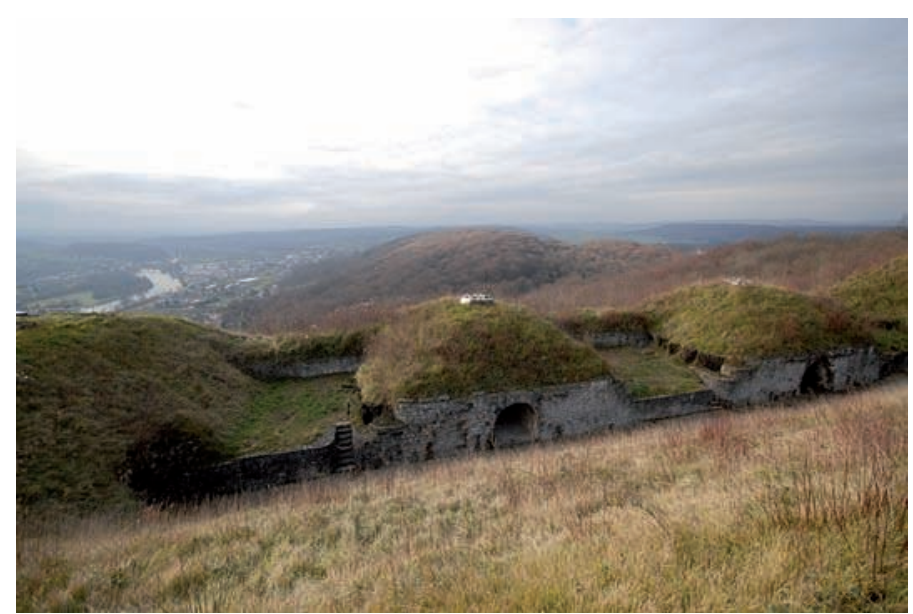

Le fort du Mont-Bart à Bavans (c) Claude Nardin 
Une deuxième antenne du CIAP pourrait trouver sa place au fort du Mont-Bart, ouvrage intégré au réseau des fortifications protégeant la frontière issu des traités de 1871 et érigé par le général Séré de Rivière. Ce fort présente un intérêt sur le plan de l'histoire et de l'architecture militaire, mais constitue également un lieu d'accueil privilégié pour une programmation grand public de spectacles vivants en période estivale. Le fort aurait donc tout naturellement vocation à accueillir cette antenne qui présenterait le Pays de Montbéliard en guerre (histoire du rattachement à la France, guerre de 1870 et guerres mondiales).

Le patrimoine industriel, intimement lié à l'histoire du pays, est extrêmement présent dans le Pays de Montbéliard : cités ouvrières, châteaux patronaux, usines... Une antenne du CIAP devrait donc traiter de l'industrialisation du Pays de Montbéliard $\left(X\right.$ III $^{\mathrm{e}}-\mathrm{XX}^{\mathrm{e}}$ : les prémices de l'industrialisation, les grandes dynasties, le paternalisme, l'origine géographique des ouvriers, les vagues migratoires, les religions du Pays de Montbéliard, l'architecture industrielle, la mémoire ouvrière). Cette antenne devrait fonctionner en étroite collaboration avec l'université Technologique de Belfort-Montbéliard, qui propose un enseignement en histoire industrielle, ainsi qu'avec les autres sites de valorisation du patrimoine industriel : musée Peugeot à Sochaux, archives Peugeot à Hérimoncourt, maison du Patrimoine à Audincourt et musée Japy de Beaucourt, qui fait actuellement l'objet de la rédaction d'un nouveau Projet Scientifique et Culturel dans la perspective d'un déménagement et agrandissement. La localisation de cette antenne pourrait être Audincourt, en raison de l'ampleur de son patrimoine industriel (lié notamment aux anciennes forges), de sa qualité de deuxième ville de l'agglomération et de la présence d'autres sites remarquables (église de l'immaculée conception et église du Sacré-Cœur), mais d'autres possibilités pourront aussi être étudiées.
Enfin, une dernière antenne serait consacrée au paysage et au patrimoine rural. Elle trouverait sa place au sein de la «Damassine », qui sera située sur la commune de Vandoncourt. Née du constat de la disparition progressive des vergers du Pays de Montbéliard, cette maison thématique a pour objectif la promotion et la protection du patrimoine fruitier. Cet équipement d'intérêt communautaire matérialisera l'engagement de l'agglomération pour la sauvegarde de ce patrimoine. Il sera la maison mère d'où rayonne l'ensemble des actions sur l'agglomération du Pays de Montbéliard, mais également un pôle exemplaire et démonstratif sur le plan environnemental et énergétique. Il abritera notamment un espace d'expositions permanentes et temporaires, des espaces réservés aux animations pédagogiques, un point de vente de produits locaux et produits liés aux fruits, un atelier de fabrication de jus de fruits pour les propriétaires de vergers désireux de produire eux-mêmes, un atelier de transformation pour fabriquer des confitures et autres préparations alimentaires à base de fruits, ainsi que l'équipe chargée de l'entretien des vergers. La présentation des aspects paysagers et d'architecture rurale (petit patrimoine, fermes...) est donc tout à fait complémentaire de la démarche déjà engagée.

\section{Un centre inséré dans un ensemble}

Ce programme ambitieux permettrait la compréhension des différentes facettes de l'évolution politique, sociale, économique et religieuse du Pays de Montbéliard, et sa traduction en termes de paysages, de développement urbain et d'architecture. Il permettrait d'autre part de donner une dimension et une cohérence accrue à l'offre touristique et culturelle sur le territoire, en favorisant une mise en réseau des sites existants et tout en sachant tirer parti de projets déjà impulsés par la communauté d'agglomération. En effet, certaines antennes seraient en fait intégrées dans des projets déjà existants, et leur appartenance
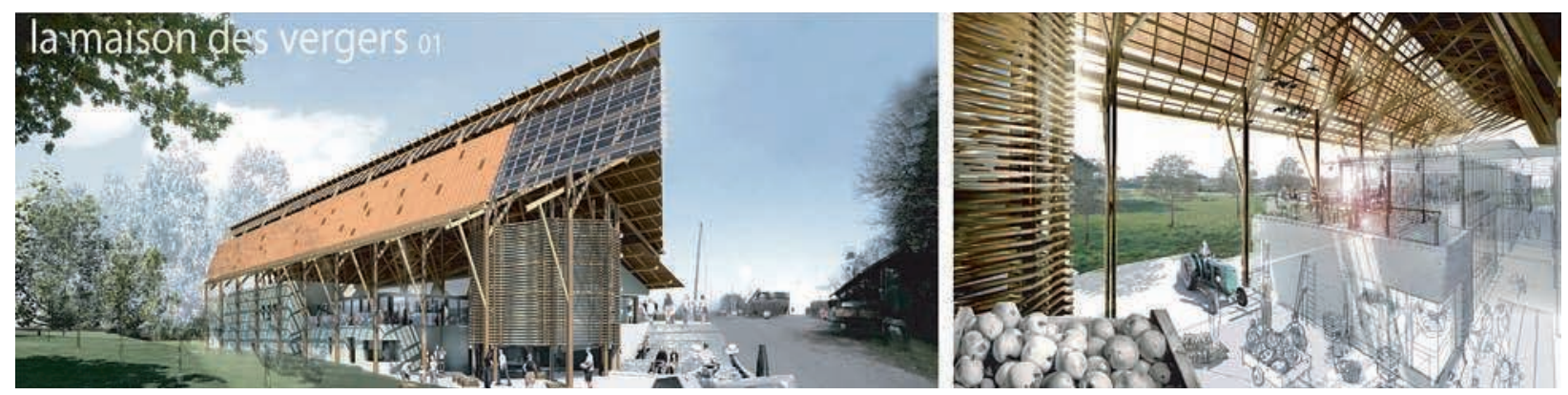

Projet de maison des vergers, dite la "Damassine » (c) Cabinet Haha architecture-Saint-Nabord 
au CIAP n'entraînerait donc pas de surcoût par rapport aux projets initiaux : valorisation touristique du fort du Mont-Bart, valorisation du théâtre de Mandeure par la construction d'un pavillon de site, maison des vergers. D'autre part, l'implantation de Montbéliard serait bien le CIAP principal : elle devrait donc pouvoir disposer de toutes les composantes demandées pour un CIAP (centre de documentation, salles d'animations pédagogiques...). Certaines antennes pourraient en revanche avoir une ampleur moindre en termes d'aménagement et de personnel. Enfin, la réalisation des différentes antennes pourrait être échelonnée dans le temps, mais dans une perspective de cohérence globale.

En résumé, la création de plusieurs antennes pour le CIAP présenterait les avantages suivants :

- elle favoriserait le déplacement des touristes vers des lieux emblématiques du Pays de Montbéliard et donc augmenterait d'autant la durée de leur séjour dans le territoire ;

- pour les habitants, elle favoriserait la mise en place d'animations de proximité ;

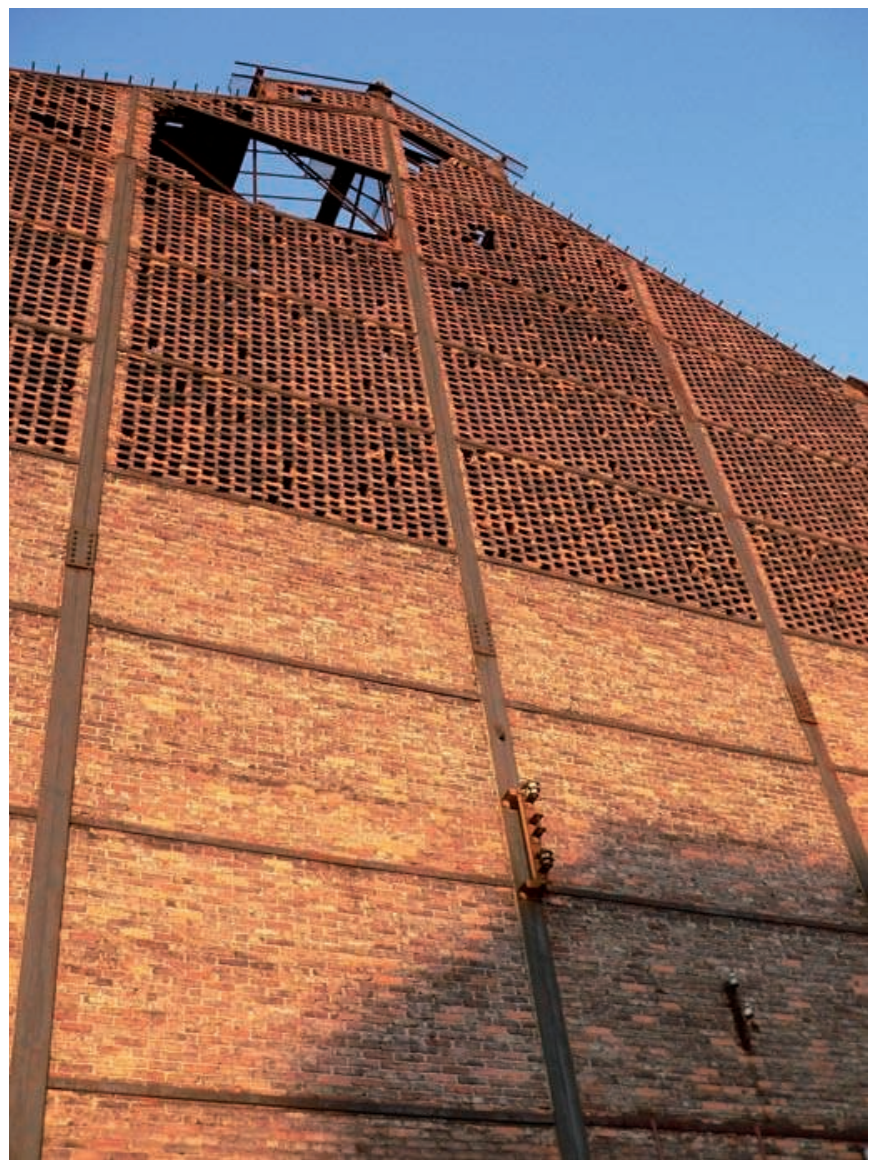

Le four Martin, site des Forges à Audincourt () Évelyne Boilaux
- elle contribuerait à créer une dynamique de mise en réseau des différents sites touristiques du pays, notamment ceux dont la communauté d'agglomération a la charge ;

- elle permettrait d'optimiser la lisibilité et la cohérence de l'offre proposée par la communauté d'agglomération du Pays de Montbéliard en termes de valorisation du patrimoine.

Cette construction intellectuelle semble globalement satisfaisante, mais diverses interrogations demeurent : quelle localisation pour le CIAP à Montbéliard et pour l'antenne sur le patrimoine industriel ? Quels moyens humains prévoir pour les CIAP ? Comment concrètement faire jouer à plein l'effet réseau entre les antennes et avec les autres structures culturelles du territoire, et notamment quelle articulation trouver avec les musées ? Une réponse aux questions précédentes devra être apportée par le Projet Scientifique et Culturel, dont un premier rendu devrait avoir lieu fin 2008, et affinée par l'étude de faisabilité du projet.

Outre les interrogations précédentes, la question la plus importante est sans doute la suivante : comment réussir à ce que l'appartenance d'un site au CIAP ne se résume pas à une pancarte « antenne CIAP » et à une exposition permanente, mais que ces lieux soient non seulement des lieux touristiques mais également des lieux que la population pourrait s'approprier ?

Au dire de collègues animateurs de l'Architecture et du Patrimoine, certains des CIAP existants sont sous-exploités, perçus comme « la chose de l'animateur » voire même considérés comme inutiles. Comment passer alors de lieux d'exposition figés à des lieux d'échange sur le patrimoine, des lieux de vie pour la population ? La solution est bien sûr à chercher en fonction des spécificités de chaque territoire. Le rayonnement du CIAP peut être facilité par le rassemblement en un même lieu du CIAP, des bureaux du service Animation du Patrimoine et des espaces d'animations. On peut aussi faire du CIAP un passage obligé ou même le point de départ des visites guidées. Les CIAP accueillent également souvent des expositions temporaires (comme à Moulins par exemple). D'autres collègues proposent des solutions plus originales : prêt de la salle d'exposition, modulable, pour des assemblées générales d'associations (Poitiers), espace consacré dans le CIAP aux zones protégées (secteur sauvegardé et ZPPAUP), avec une présentation de ces zones, des démarches à entreprendre selon les types de demande, des fiches techniques 
sur les modes d'intervention et des plages horaires de présence de l'architecte conseil de la ville et de l'architecte des Bâtiments de France pour répondre aux questions des habitants (Chambéry).

\section{Une réponse à inventer en fonction de chaque situation}

La bonne formule pour le Pays de Montbéliard est à inventer. Pour tenter d'y répondre, le service Animation du Patrimoine du Pays de Montbéliard a proposé aux élus communautaires de s'engager dans une forme de concertation publique, qui aurait comme objectifs de permettre d'enrichir le Projet Scientifique et Culturel, d'identifier les moyens permettant l'appropriation maximale du CIAP par les habitants sur le long terme, et de trouver un nom porteur et fédérateur pour la structure, « centre d'interprétation de l'Architecture et du Patrimoine » n'étant pas forcément le plus adapté...

Cette phase contribuerait à une meilleure appropriation par la population de ce projet, y compris les acteurs associatifs et les élus : chaque personne souhaitant s'investir pourrait ainsi y contribuer pendant une période déterminée par la communauté d'agglomération. La population pourrait ainsi s'exprimer sur le discours à tenir dans ce CIAP (quel patrimoine pour le Pays de Montbéliard ? Quels éléments mettre en avant ?), mais aussi apporter sa contribution en terme de témoignages, documents, qui pourraient être ensuite valorisés par le service Animation, dans les expositions permanentes ou dans d'autres cadres, l'aspect humain et mémoriel devant nécessairement apparaître en contrepoint du discours historique et architectural. Nous espérons également par cette démarche permettre une collaboration étroite avec les associations œuvrant dans le domaine du patrimoine et tous les érudits ou personnes ressources locales qui n'auraient pas été identifiées par le service Animation du Patrimoine. Cette phase d'élaboration du projet serait bien entendu suivie par des études de faisabilité et programmation puis par une réalisation concrète avec un comité plus restreint : villes concernées et structures et associations œuvrant dans le domaine du patrimoine sur le territoire, sous la responsabilité scientifique du comité scientifique du service Animation du Patrimoine.

Nous profitons de cette contribution pour lancer un appel aux chercheurs qui souhaiteraient s'associer à notre réflexion pour la conception de ce CIAP, afin, nous le souhaitons, qu'il puisse être exemplaire dans sa conception comme dans sa réalisation et quiil joue pleinement son rôle pour les habitants du Pays de Montbéliard comme pour les touristes que nous invitons nombreux sur le territoire.

\section{Notes}

(1) Centre d'Interprétation de l'Architecture et du Patrimoine mode d'emploi. Ministère de la Culture et de la Communication, 2004.

(2) Ou « métropole d'Art et d'Histoire ", nouvelle appellation revendiquée par certaines communautés d'agglomération gérant l'animation du patrimoine, comme celle de Rennes. 